Received: 31 May 2017

Accepted: 22 November 2017

Published online: 05 December 2017

\section{Preoperative serum thyroglobulin predicts initial distant metastasis in patients with differentiated thyroid}

\section{cancer}

Hosu Kim ${ }^{1,2}$, Young Nam Kim ${ }^{1}$, Hye In Kim ${ }^{1}$, So Young Park ${ }^{1}$, Jun-Ho Choe ${ }^{3}$, Jung-Han Kim³, Jee Soo Kim ${ }^{3}$, Jae Hoon Chung ${ }^{1}$, Tae Hyuk Kim ${ }^{1}{ }^{1}$ \& Sun Wook Kim ${ }^{1}$

Differentiated thyroid cancer (DTC) generally has a favorable prognosis. However, a small percentage of patients suffer from initial distant metastasis (DM). To date, there is no effective predictor for the presence of initial DM. The aim of this study was to determine if preoperative serum thyroglobulin (Tg) level could predict initial DM in DTC. We reviewed an institutional thyroid cancer database from October 1994 to February 2016. To determine the Tg cutoff for predicting initial DM, 4,735 patients who were diagnosed with DTC were included in this study. Fifty-seven patients $(1.2 \%)$ were identified as having DTC with initial DM. Median preoperative Tg level was $328.4 \mathrm{ng} / \mathrm{ml}$ in the initial DM group and $10.0 \mathrm{ng} / \mathrm{ml}$ in the non-DM group. Initial DM was the most important factor affecting serum Tg level $(\beta=2,049.32 \pm 103.40 ; P<0.001)$. The Tg cutoff level that distinguished overall DM with the greatest accuracy was $63.4 \mathrm{ng} / \mathrm{ml}$ [area under the ROC curve 0.914 , sensitivity $84.2 \%$, specificity $90.6 \%$, negative likelihood ratio (LR) 0.17 , and positive LR 8.97]. Preoperative Tg levels were useful for predicting initial DM of DTC. Measurement of serum Tg in patients with DTC may guide preoperative staging evaluation and initial treatment.

Most patients with differentiated thyroid cancer (DTC) have a favorable prognosis and initial distant metastasis $(\mathrm{DM})$ is rare ${ }^{1,2}$. However, the mortality rate significantly increases when initial DM is present ${ }^{3-7}$. Therefore, early diagnosis and treatment of initial DM is important for the treatment of DTC patients ${ }^{8,9}$. For medullary thyroid carcinoma, serum calcitonin levels predict initial DM, recurrence and prognosis, but DTC has no available predictor $^{10,11}$.

Thyroglobulin (Tg) is a glycoprotein that is secreted from follicular cells of the thyroid gland. Therefore $\mathrm{Tg}$ reflects the burden of the follicular cell, whether it is benign or malignant ${ }^{12}$. Tg helps predict DTC recurrence in a postoperative setting, but its usefulness for preoperative diagnosis is less clear ${ }^{13-19}$. Tg levels can rise when follicular cells increase, such as in goiter and thyroiditis, even if they are not indicative of pathological status ${ }^{20}$. However, in patients with a high tumor burden, such as patients with initial DM, serum Tg concentration may increase unequivocally ${ }^{8,21-23}$.

The purpose of this study was to determine the correlation between preoperative serum $\mathrm{Tg}$ concentration and the existence of distant metastasis in DTC patients. We evaluated whether preoperative serum Tg concentration could be used as a predictive marker for initial DM in clinical settings.

\section{Results}

Differences between presence of initial DM and non-DM group. Of the 4,735 enrolled patients, 57 had initial DM. No difference was observed in age and sex between the initial DM and non-DM group. By tumor histology, follicular thyroid carcinoma (FTC) was more frequent in the initial DM group than the non-DM group $(35.1 \%$

${ }^{1}$ Division of Endocrinology \& Metabolism, Department of Medicine, Thyroid Center, Samsung Medical Center, Sungkyunkwan University School of Medicine, Seoul, Korea. 'Division of Endocrinology, Department of Medicine, Gyeongsang National University Changwon Hospital, Changwon, Korea. ${ }^{3}$ Division of Breast and Endocrine Surgery, Department of Surgery, Samsung Medical Center, Sungkyunkwan University School of Medicine, Seoul, Korea. Correspondence and requests for materials should be addressed to T.H.K. (email: taehyukmd.kim@samsung.com) or S.W.K. (email: swkimmd@skku.edu) 


\begin{tabular}{|l|l|l|l|}
\hline Characteristics & $\begin{array}{l}\text { Initial DM group } \\
(\boldsymbol{n}=\mathbf{5 7})\end{array}$ & $\begin{array}{l}\text { Non-DM group } \\
(\boldsymbol{n}=\mathbf{4 6 7 8})\end{array}$ & P value \\
\hline Age at diagnosis (years) & $47.75 \pm 17.58$ & $46.36 \pm 11.24$ & 0.555 \\
\hline Sex (female) & $42(73.7 \%)$ & $3670(78.5 \%)$ & 0.417 \\
\hline RAI treatment & $55(96.5 \%)$ & $3003(64.2 \%)$ & $<\mathbf{0 . 0 0 1}$ \\
\hline Initial CND & $44(77.2 \%)$ & $3992(85.3 \%)$ & 0.091 \\
\hline Tumor histology & & & $<\mathbf{0 . 0 0 1}$ \\
\hline PTC & $37(64.9 \%)$ & $4585(98.0 \%)$ & \\
\hline FTC & $20(35.1 \%)$ & $93(2.0 \%)$ & \\
\hline Tumor size (cm) & $3.19 \pm 1.94$ & $1.04 \pm 1.02$ & $<\mathbf{0 . 0 0 1}$ \\
\hline Multifocal tumor (tumor number) & $1.57 \pm 1.05$ & $1.51 \pm 1.01$ & 0.633 \\
\hline Positive gross ETE & $19(33.3 \%)$ & $568(12.1 \%)$ & $<\mathbf{0 . 0 0 1}$ \\
\hline Positive resection margin & $13(22.8 \%)$ & $211(4.5 \%)$ & $<\mathbf{0 . 0 0 1}$ \\
\hline LNM $>5$ & $26(54.6 \%)$ & $503(10.8 \%)$ & $<\mathbf{0 . 0 0 1}$ \\
\hline Positive lymphatic invasion & $4(7.0 \%)$ & $57(1.2 \%)$ & $\mathbf{0 . 0 0 6}$ \\
\hline Positive blood vessel invasion & $17(29.8 \%)$ & $75(1.6 \%)$ & $<\mathbf{0 . 0 0 1}$ \\
\hline Preoperative Tg concentration $(\mathrm{ng} / \mathrm{ml})$ & $328.4(2.3-42680.0)$ & $10.0(0.1-6542.0)$ & $\mathbf{0 . 0 0 6}$ \\
\hline
\end{tabular}

Table 1. Differences between initial DM and non-DM groups. Continuous data were given as mean \pm SD; categorical data were given as absolute numbers (percentage). Preoperative Tg concentration was presented as median (range). $\chi 2$-test or Fisher's exact test were used for categorical data, and t-tests or the Mann-Whitney test for continuous data. DM, distant metastases; RAI, radioactive iodine; CND, central neck dissection; PTC, papillary thyroid carcinoma; FTC, follicular thyroid carcinoma; ETE, extrathyroidal extension; LNM, lymph node metastases; Tg, thyroglobulin.

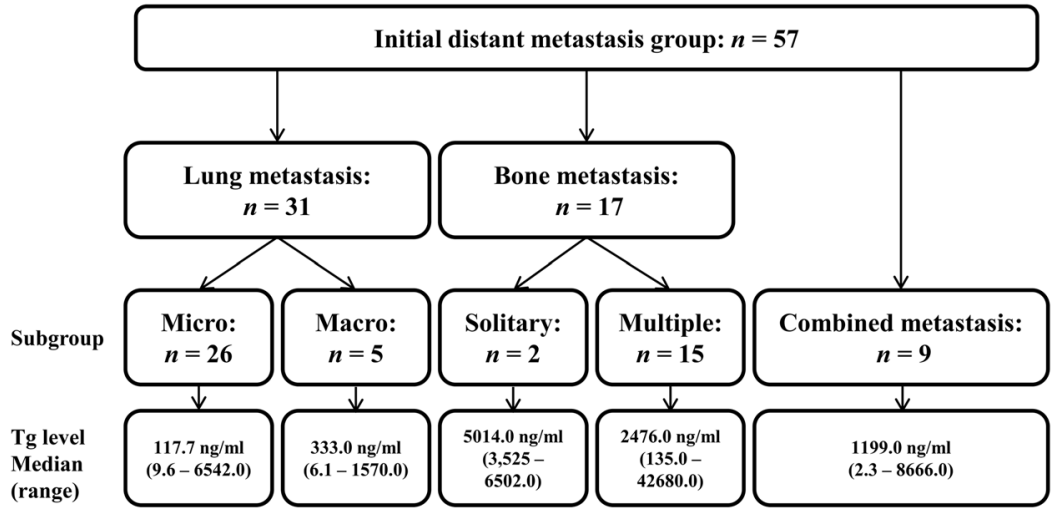

Figure 1. Flow diagram of the study. Fifty-seven patients were diagnosed with initial distant metastasis (DM). Patients were classified according to site of distant metastasis. Diagnoses were 31 with lung metastasis, 17 with bone metastasis, and 9 with combined lung/bone metastasis. Lung metastasis were subdivided into micronodular and macronodular. Bone metastasis were subdivided into solitary and multiple.

vs. $2.0 \%, \mathrm{P}<0.001)$. Mean tumor size was $3.19 \mathrm{~cm}$ in the initial DM group, which was larger than the non-DM group. However, multifocal tumor burden was not significantly different between the two groups. Gross extrathyroidal extension (ETE), lymphatic invasion, blood vessel invasion, positive resection margin and lymph node metastasis $(\mathrm{LNM})>5$ were all significantly higher in the initial DM group than the non-DM group (Table 1).

Characteristics of patients with initial distant metastasis. Overall, 57 patients were diagnosed with initial DM: 37 with papillary thyroid carcinoma (PTC) and 20 with FTC. In addition, 26 patients (45.6\%) were classified as having micronodular lung metastasis, and 5 (8.8\%) with macronodular lung metastasis; two patients (3.5\%) were classified as having single bone metastasis, and $15(26.3 \%)$ with multiple bone metastases. Nine patients (15.8\%) had combined lung and bone metastasis. Most lung metastasis were PTC $(n=29)$, with two that were FTC. Also, 13 FTC patients were diagnosed as having bone metastasis and 4 were diagnosed as having bone metastasis from PTC (Fig. 1).

Micronodular lung metastasis was associated with the lowest preoperative serum Tg, with a median of $117.7 \mathrm{ng} /$ $\mathrm{ml}$ (range 9.6-6,542.0 ng/ml) among initial DM subgroups. Macronodular lung metastasis had a preoperative serum $\mathrm{Tg}$ median of $333.0 \mathrm{ng} / \mathrm{ml}$ (range 6.1-1,570.0 ng/ml). Preoperative serum Tg was significantly higher when bone metastasis was present. Preoperative serum Tg for solitary bone metastasis had a median of 5,014.0 ng/ml (range 3,525.0-6,502.0 ng/ml), multiple bone metastasis had a median of 2,476.0 ng/ml (range 135.0-42,680.0 ng/ml), and combined metastasis had a median of $1,199.0 \mathrm{ng} / \mathrm{ml}$ (range 2.3-8,666.0 ng/ml) (Fig. 1). 


\begin{tabular}{|c|c|c|c|c|}
\hline \multirow[b]{2}{*}{ Characteristics } & \multicolumn{2}{|l|}{ Unadjusted } & \multicolumn{2}{|l|}{ Adjusted } \\
\hline & $\beta \pm \mathrm{SE}$ & $P$ value & $\beta \pm \mathrm{SE}$ & $P$ value \\
\hline Age at diagnosis (years) & $0.75 \pm 1.02$ & 0.460 & - & - \\
\hline Sex (female) & $11.69 \pm 28.09$ & 0.677 & - & - \\
\hline Tumor histology (FTC) & $1253.39 \pm 73.52$ & $<0.001$ & $662.93 \pm 80.13$ & $<0.001$ \\
\hline Tumor size & $12.20 \pm 10.71$ & $<0.001$ & $48.82 \pm 10.86$ & $<0.001$ \\
\hline Multifocal tumor (tumor number) & $-6.12 \pm 11.40$ & 0.591 & - & - \\
\hline Positive lymphatic invasion & $74.57 \pm 102.51$ & 0.467 & - & - \\
\hline Positive blood vessel invasion & $1135.89 \pm 82.11$ & $<0.001$ & $378.59 \pm 86.97$ & $<0.001$ \\
\hline Positive resection margin & $59.62 \pm 54.45$ & 0.274 & - & - \\
\hline $\mathrm{LNM}>5$ & $39.74 \pm 36.69$ & 0.279 & $-70.19 \pm 35.04$ & 0.045 \\
\hline Positive gross ETE & $19.97 \pm 35.08$ & 0.569 & - & - \\
\hline Presence of initial DM & $2456.00 \pm 99.82$ & $<0.001$ & $2049.32 \pm 103.40$ & $<0.001$ \\
\hline
\end{tabular}

Table 2. Factors associated with preoperative serum Tg elevation. Linear regression analysis was performed. SE, standard error; FTC, follicular thyroid carcinoma; ETE, extrathyroidal extension; LNM, lymph node metastases; DM, distant metastases.

Factors associated with preoperative serum Tg elevation. Preoperative serum $\mathrm{Tg}$ concentration was higher in the initial DM group than the non-DM group. Tg concentration for the initial DM group was a median of $328.4 \mathrm{ng} / \mathrm{ml}$ (range 2.3-42,680.0 ng/ml) compared to the non-DM group with a median of $10.0 \mathrm{ng} / \mathrm{ml}(\mathrm{range}$ $0.1-6,542.0 \mathrm{ng} / \mathrm{ml}$ ) (Table 1). Preoperative serum Tg concentration for patients with FTC was significantly higher than for patients with PTC (median $87.6 \mathrm{ng} / \mathrm{ml}$, range $0.5-42,680.0$ for FTC and $9.9 \mathrm{ng} / \mathrm{ml}$, range $0.1-8666.0$ for PTC, $P<0.001)$. Preoperative serum Tg concentration was higher for large tumors $(\beta=12.20, P<0.001)$ and blood vessel invasion (median $51.9 \mathrm{ng} / \mathrm{ml}$, range $0.8-22,666.0$ for positive blood vessel invasion and median $10.0 \mathrm{ng} / \mathrm{ml}$, range $0.1-42,680.0$ for negative blood vessel invasion). Multivariate linear regression analysis showed only FTC, large tumor size, blood vessel invasion, $\mathrm{LNM}>5$ and initial DM were significantly associated with preoperative serum Tg concentration ( $\beta=662.93$ and $P<0.001$ for FTC, $\beta=48.82$ and $P<0.001$ for tumor size, $\beta=378.59$ and $P<0.001$ for blood vessel invasion, $\beta=-70.19$ and $P=0.045$ for $\mathrm{LNM}>5, \beta=2049.32$ and $P<0.001$ for initial DM) (Table 2).

ROC analysis for identifying initial distant metastasis. In ROC analysis, the optimal cutoff for predicting overall DM was $63.4 \mathrm{ng} / \mathrm{ml}$ with area under the ROC curve of 0.914 and corresponding sensitivity $84.2 \%$, specificity 90.6\%, negative likelihood ratios (LR) 0.17, and positive LR 8.97 (Fig. 2). Negative predictive value (NPV) was $96.4 \%$ and positive predictive value (PPV) was $9.29 \%$ in our cohort. When study populations were stratified by tumor histology, the optimal cutoff for PTC to predict initial DM was $63.4 \mathrm{ng} / \mathrm{ml}$ and $1192.0 \mathrm{ng} / \mathrm{ml}$ for FTC (sensitivity 75.7\%, specificity $91.4 \%$ for PTC; sensitivity $75.0 \%$ and specificity $92.5 \%$ for FTC).

Univariate and multivariate logistic regression analyses. Clinical characteristics including preoperative serum $\mathrm{Tg}(>63.4 \mathrm{ng} / \mathrm{ml}$ or $\leq 63.4 \mathrm{ng} / \mathrm{ml}$ ), age at diagnosis, sex (male or female), tumor size, multifocal tumor, lymphatic invasion, blood vessel invasion, positive resection margin, $\mathrm{LNM}>5$ and presence of gross ETE were analyzed as independent variables using logistic regression analyses (Table 3). In univariate logistic regression analyses, preoperative serum $\mathrm{Tg}>63.4$ (odds ratio [OR], 51.50; 95\% confidence interval [CI], 25.10-105.66; $P<0.001$ ), tumor size (OR, 1.90; 95\% CI, 1.66-2.17; $P<0.001$ ), lymphatic invasion (OR, 6.12; 95\% CI, 2.14$17.47 ; P=0.001)$, blood vessel invasion (OR, 26.08; 95\% CI, 14.05-48.08; $P<0.001)$, positive resection margin (OR, 6.26; 95\% CI, 3.32-11.79; $P<0.001$ ), LNM $>5$ (OR, 6.69; 95\% CI, 4.10-11.82; $P<0.001$ ), and presence of gross ETE (OR, 3.62; 95\% CI, 2.07-6.32; $P<0.001$ ) were significant factors that distinguished between the initial $\mathrm{DM}$ and non-DM groups. In multivariate logistic regression analyses, preoperative serum $\mathrm{Tg}(\mathrm{OR}, 24.62 ; 95 \% \mathrm{CI}$, $11.59-52.28 ; P<0.001$ ), age at diagnosis (OR, $1.03 ; 95 \% \mathrm{CI}, 1.01-1.05 ; P=0.015)$, tumor size (OR, $1.11 ; 95 \% \mathrm{CI}$, 1.08-1.30; $P<0.001$ ), blood vessel invasion (OR, 8.06; 95\% CI, 3.78-17.17; $P<0.001)$, positive resection margin (OR, 4.01; 95\% CI, 1.84-8.76; P $<0.001$ ), and LNM $>5$ (OR, 4.08; 95\% CI, 2.14-7.79; $P<0.001$ ) were independent predictive factors for differentiating between the initial DM and non-DM groups.

\section{Discussion}

This study demonstrated that preoperative serum Tg concentration well predicted initial DM in DTC patients. We also proposed a specific Tg cutoff value of $63.4 \mathrm{ng} / \mathrm{ml}$ as an optimal threshold with the greatest accuracy for practical use in clinics.

Previous studies have used preoperative serum Tg for differential diagnosis of DTC. Petric et al. differentiated between follicular adenoma and carcinoma, and between follicular carcinoma and Hurthle cell carcinoma using preoperative serum $\mathrm{Tg}^{18,24}$. Sarah et al. showed that preoperative serum $\mathrm{Tg}$ concentration was significantly higher with initial DM. However, a cutoff for serum Tg was not presented ${ }^{22,25}$. Some studies found that preoperative serum Tg concentrations are not useful for differential diagnosis of $\mathrm{DTC}^{15,19}$. The reason for this may be the many factors that affect preoperative serum Tg concentration ${ }^{20}$. Therefore, in this study, we analyzed factors affecting preoperative Tg and set an optimal cutoff for preoperative serum Tg in situations such as initial DM in which Tg concentration is unequivocally raised due to a high tumor burden. 


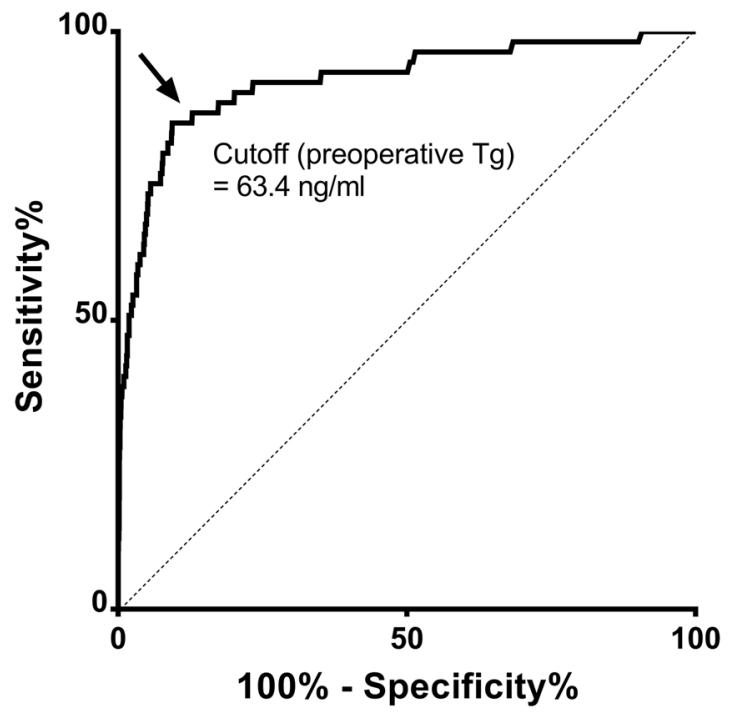

Figure 2. ROC curves for differentiating between the presence of initial distant metastases and non-distant metastases groups. Area under the ROC curve (preoperative serum Tg), 0.914 (95\% CI, 0.869-0.960). Cutoff (preoperative serum Tg), $63.4 \mathrm{ng} / \mathrm{ml}$; sensitivity, $84.2 \%$; specificity, 90.6\%; positive LR, 8.97; negative LR, 0.17; PPV, 9.3\%; NPV, 96.4\%.

\begin{tabular}{|l|l|l|l|l|}
\hline \multirow{2}{*}{ Characteristics } & \multicolumn{2}{l}{ Unadjusted } & \multicolumn{2}{l|}{ Adjusted } \\
\cline { 2 - 5 } & Odds ratio (95\% CI) & $\boldsymbol{P}$ value & Odds ratio (95\% CI) & P value \\
\hline Age at diagnosis (years) & $1.01(0.99-1.03)$ & 0.359 & $1.03(1.01-1.05)$ & $\mathbf{0 . 0 1 5}$ \\
\hline Sex (female) & $0.77(0.43-1.39)$ & 0.386 & & \\
\hline Tumor histology (FTC) & $26.65(14.90-47.66)$ & $<\mathbf{0 . 0 0 1}$ & & \\
\hline Tumor size & $1.90(1.66-2.17)$ & $<\mathbf{0 . 0 0 1}$ & $1.11(1.08-1.30)$ & $<\mathbf{0 . 0 0 1}$ \\
\hline Multifocal tumor(tumor number) & $1.06(0.84-1.33)$ & 0.633 & & \\
\hline Positive lymphatic invasion & $6.12(2.14-17.47)$ & $\mathbf{0 . 0 0 1}$ & & $<\mathbf{0 . 0 0 1}$ \\
\hline Positive blood vessel invasion & $26.08(14.05-48.08)$ & $<\mathbf{0 . 0 0 1}$ & $8.06(3.78-17.17)$ & $<\mathbf{0 . 0 0 1}$ \\
\hline Positive resection margin & $6.26(3.32-11.79)$ & $<\mathbf{0 . 0 0 1}$ & $4.01(1.84-8.76)$ & $<\mathbf{0 . 0 0 1}$ \\
\hline LNM $>5$ & $6.69(4.10-11.82)$ & $<\mathbf{0 . 0 0 1}$ & $4.08(2.14-7.79)$ & \\
\hline Positive gross ETE & $3.62(2.07-6.32)$ & $<\mathbf{0 . 0 0 1}$ & & $<\mathbf{0 . 0 0 1}$ \\
\hline Tg 63.4 & $51.50(25.10-105.66)$ & $<\mathbf{0 . 0 0 1}$ & $24.62(11.59-52.28)$ & \\
\hline
\end{tabular}

Table 3. Logistic regression analyses of initial distant metastases according to clinicopathological factors. Logistic regression analysis was performed. CI, confidence interval; FTC, follicular thyroid carcinoma; ETE, extra-thyroidal extension; LNM, lymph node metastases; Tg, thyroglobulin.

In our study, tumor histology, tumor size, blood vessel invasion, LNM $>5$ and initial DM were significant factors that increased preoperative serum Tg concentration (Table 2). Among these factors, initial DM was the most significant for increased preoperative serum Tg. In multivariate linear regression analysis, the $\beta$ value for initial DM was 3.1 times higher than for tumor histology and 42.7 times higher than for tumor size. Based on ROC curves, the preoperative Tg concentration that predicted initial DM was $63.4 \mathrm{ng} / \mathrm{ml}$. In multivariate logistic regression analysis, the odds ratio for a Tg cutoff of $63.4 \mathrm{ng} / \mathrm{ml}$ as a predictor of initial DM was 24.62 and the effect size was significantly higher than for age at diagnosis, tumor size, blood vessel invasion, resection margin or LNM. Therefore, preoperative serum Tg may be a strong indicator of initial DM from DTC. If the preoperative serum Tg cutoff of $63.4 \mathrm{ng} / \mathrm{ml}$ were applied, $84.2 \%$ (48/57) of initial DM patients in our study could have been found at initial workup. If preoperative serum $\mathrm{Tg}$ is exceedingly high, clinicians should discuss with patients the possibility of bone metastasis from DTC and appropriate imaging studies in addition to chest CT scans should be pursued.

We found some discrepancies between preoperative serum Tg concentration and initial DM. When reviewing 9 patients with initial DM but preoperative serum Tg lower than $63.4 \mathrm{ng} / \mathrm{ml}, 7 \mathrm{had}$ micronodular lung metastasis, 1 had macronodular lung metastasis and 1 had combined metastasis. These 9 patients were all PTC and had no blood vessel invasion. Of these patients, 1 patient who had combined metastasis and 1 patient with micronodular lung metastasis did not respond to radioactive iodine (RAI) therapy and died. Since these 2 patients showed a lack of RAI avidity in the metastatic lesions, we speculated that the patients did not develop an elevated Tg because of tumor dedifferentiation. Another 6 patients with micronodular lung metastasis entered remission or maintained a stable disease status after RAI therapy. They did not develop an elevated Tg. This can be attributed to a 
subclinical burden of microscopic metastases, which was insufficient to produce a significant amount of Tg. Also, the remaining patient with macronodular lung metastasis had a solitary lesion. Therefore, the authors speculate that if the burden of metastasis is too small or localized in a solitary site, serum Tg may not rise to sufficient levels to promote suspicion of initial DM.

In addition, in the non-DM group, we reviewed 34 patients with preoperative serum $\mathrm{Tg}$ who had a preoperative serum Tg greater than $1000 \mathrm{ng} / \mathrm{ml}$. One had a large goiter, one had a multifocal PTC of more than 10, and one had a coexisting $8 \mathrm{~cm}$ follicular adenoma other than PTC. The remaining 31 patients had hypervascular tumors that were large enough to occupy the thyroid gland.

Savvy practitioners may use an alternative Tg cutoff derived from LRs. When preoperative serum $\mathrm{Tg}$ was more than $81.2 \mathrm{ng} / \mathrm{ml}$, positive LR was 10.05 , which was above 10 for the first time (sensitivity $75.4 \%$ and specificity $92.5 \%$ ). NPV was $95.9 \%$ and PPV was $7.4 \%$. A positive LR greater than 10 means that a positive test is good at ruling in a diagnosis ${ }^{26}$. NPV was high due to a low prevalence of initial DM. Therefore, it is also a good idea to make conservative decisions using a cutoff based on higher preoperative serum $\operatorname{Tg}(\mathrm{LR}>10)$ instead of an accuracy-derived cutoff ${ }^{27}$.

The strength of our study is the relatively large number of patients with initial $\mathrm{DM}^{28}$. Also, we proposed an optimal preoperative serum $\mathrm{Tg}$ concentration cutoff for practical use in clinics. In addition, we presented clinicopathological factors that may lead to elevation of serum Tg levels of DTC patients in the preoperative state. A limitation of our study was the retrospective design. Also, although our study included a relatively large number of patients with initial DM, patients were recruited from a single teaching hospital and the population was therefore prone to selection bias. Because of the low prevalence of initial DM in DTC patients, a multicenter prospective study may be needed to validate the generalizability of our findings.

In conclusion, preoperative serum Tg concentration is a useful marker for predicting the presence of initial DM from DTC, despite the presence of other interfering factors. Measurement of serum Tg in patients with biopsy-proven DTC may guide preoperative staging evaluation and initial treatment.

\section{Patients and Methods}

The Institutional Review Board at Samsung Medical Center approved this study (IRB File No. 2017-02-056). We retrospectively screened a prospectively maintained institutional thyroid cancer database of patients who were diagnosed with and treated for DTC at Samsung Medical Center between 1994 and 2016. The final cohort was 4,735 DTC patients whose preoperative serum Tg concentrations were available for analysis. Patients were a median of 47.0 years old with $78.4 \%$ women. Total thyroidectomy was performed on $77.1 \%$ and RAItherapy was given to $64.6 \%$. PTC was diagnosed in $97.6 \%$ and FTC in $2.4 \%$ of patients. Mean follow-up was 7.0 years. Pathological T stage was T1 in $41.9 \%$, T2 in $2.9 \%$, T3 in 51.6\%, and T4 in 3.6\%. Pathological N stage was $31.9 \%$ $\mathrm{N} 1 \mathrm{a}$ and $9.7 \% \mathrm{~N} 1 \mathrm{~b}$. Of the patients, 57 were diagnosed with initial DM. Initial DM was defined as a suspicious lesion on pathology and/or imaging such as a whole body scan, computed tomography (CT), magnetic resonance imaging, or positron emission tomography before surgery or within 6 months after surgery. In cases of ambiguity on imaging, a DM diagnosis was established by medical record review up to 6 months after surgery. For analysis, we classified patients according to site, size and number of initial DMs. First, DM was classified as lung or bone. Lung metastases were subdivided into micronodular lung metastases smaller than $1 \mathrm{~cm}$ and macronodular lung metastases larger than $1 \mathrm{~cm}$ on image study. Bone metastases were classified as solitary or multiple bone metastases. DM involving more than two organs was classified as combined metastasis. In our study, initial DM was not diagnosed at sites other than lung and bone $e^{5,23,28}$.

Serum Tg concentration was measured using immunoradiometric assays with a BRAHMS Tg plus RIA assay (BRAHMS, Henningsdoft, Germany). The functional sensitivity of the Tg plus RIA assay was $0.2 \mathrm{ng} / \mathrm{ml}$, and analytical sensitivity was $0.008 \mathrm{ng} / \mathrm{ml}$. Intraassay coefficient of variation $(\mathrm{CV})$ was $3.4 \%$ and interassay CV was $4.9 \%^{29}$. Several factors were included in the analyses to evaluate relationships with serum Tg concentration. Age at diagnosis and sex were included as patient factors. Tumor size, multifocal tumor (tumor number), LNM, lymphatic invasion, vessel invasion, ETE, and resection margin were tumor factors. The patients with anti-Tg antibodies positive $(>100 \mathrm{IU} / \mathrm{mL})$ were excluded from the study (three patients in the initial DM group were excluded).

Statistical analysis. Continuous data were expressed as mean \pm standard deviation (SD). Data on categorical characteristics were expressed as percent values or absolute numbers. Preoperative Tg concentration was presented as median (range). For comparisons of clinical and pathological characteristics between the initial DM and non-DM groups, the $\chi^{2}$-test or Fisher's exact test were used for categorical data, and $t$-tests or the Mann-Whitney test were used for continuous data. Multivariate linear regression analysis was used to analyze factors that affected preoperative serum Tg elevation. Receiver operative characteristic (ROC) curve analysis was used to determine the cutoff level for preoperative serum Tg to distinguish patients with initial DM. Sensitivity, specificity, and positive and negative LRs were calculated using an established method ${ }^{26,30}$. Pretest probability was $1.2 \%$ based on the average incidence of initial DM in the general population and in our data ${ }^{1}$. LRs represented how many times more (or less) frequently patients with the disease presented with a particular result compared to patients without the disease. LRs $>10$ or $<0.1$ were considered strong evidence to confirm or exclude initial DM, respectively. $P<0.05$ was considered significant. Statistical analysis was performed using SPSS software version 23 (SPSS Inc., Chicago, IL, USA).

Data availability. The datasets generated during and/or analyzed during the current study are available from the corresponding author on reasonable request. 


\section{References}

1. Hoie, J., Stenwig, A. E., Kullmann, G. \& Lindegaard, M. Distant metastases in papillary thyroid cancer. A review of 91 patients. Cancer 61, 1-6 (1988).

2. Shaha, A. R., Shah, J. P. \& Loree, T. R. Differentiated thyroid cancer presenting initially with distant metastasis. Am J Surg 174, 474-476 (1997).

3. Beierwaltes, W. H., Nishiyama, R. H., Thompson, N. W., Copp, J. E. \& Kubo, A. Survival time and "cure" in papillary and follicular thyroid carcinoma with distant metastases: statistics following University of Michigan therapy. J Nucl Med 23, 561-568 (1982).

4. Choi, Y. M. et al. Standardized Thyroid Cancer Mortality in Korea between 1985 and 2010. Endocrinol Metab (Seoul) 29, 530-535 (2014).

5. Massin, J. P. et al. Pulmonary metastases in differentiated thyroid carcinoma. Study of 58 cases with implications for the primary tumor treatment. Cancer 53, 982-992 (1984).

6. Pittas, A. G. et al. Bone metastases from thyroid carcinoma: clinical characteristics and prognostic variables in one hundred forty-six patients. Thyroid 10, 261-268 (2000).

7. Schlumberger, M. et al. Long-term results of treatment of 283 patients with lung and bone metastases from differentiated thyroid carcinoma. J Clin Endocrinol Metab 63, 960-967 (1986).

8. Lee, J. \& Soh, E. Y. Differentiated thyroid carcinoma presenting with distant metastasis at initial diagnosis clinical outcomes and prognostic factors. Ann Surg 251, 114-119 (2010).

9. Sampson, E., Brierley, J. D., Le, L. W., Rotstein, L. \& Tsang, R. W. Clinical management and outcome of papillary and follicular (differentiated) thyroid cancer presenting with distant metastasis at diagnosis. Cancer 110, 1451-1456 (2007).

10. Cho, Y. Y. et al. Clinical outcomes of patients with hypercalcitoninemia after initial treatment for medullary thyroid cancer and postoperative serum calcitonin cutoffs for predicting structural recurrence. Head Neck 38, 1501-1508 (2016).

11. Hadoux, J., Pacini, F., Tuttle, R. M. \& Schlumberger, M. Management of advanced medullary thyroid cancer. Lancet Diabetes Endocrinol 4, 64-71 (2016).

12. Whitley, R. J. \& Ain, K. B. Thyroglobulin: a specific serum marker for the management of thyroid carcinoma. Clin Lab Med 24, 29-47 (2004).

13. Besic, N., Sesek, M., Peric, B., Zgajnar, J. \& Hocevar, M. Predictive factors of carcinoma in 327 patients with follicular neoplasm of the thyroid. Med Sci Monit 14, CR459-467 (2008).

14. Giovanella, L., Ceriani, L., Ghelfo, A., Maffioli, M. \& Keller, F. Preoperative undetectable serum thyroglobulin in differentiated thyroid carcinoma: incidence, causes and management strategy. Clin Endocrinol (Oxf) 67, 547-551 (2007).

15. Guarino, E. et al. Presurgical serum thyroglobulin has no prognostic value in papillary thyroid cancer. Thyroid 15, 1041-1045 (2005).

16. Lee, E. K. et al. Preoperative serum thyroglobulin as a useful predictive marker to differentiate follicular thyroid cancer from benign nodules in indeterminate nodules. J Korean Med Sci 27, 1014-1018 (2012).

17. Mazzaferri, E. L. et al. A consensus report of the role of serum thyroglobulin as a monitoring method for low-risk patients with papillary thyroid carcinoma. J Clin Endocrinol Metab 88, 1433-1441 (2003).

18. Petric, R., Perhavec, A., Gazic, B. \& Besic, N. Preoperative serum thyroglobulin concentration is an independent predictive factor of malignancy in follicular neoplasms of the thyroid gland. J Surg Oncol 105, 351-356 (2012).

19. Suh, I. et al. Serum thyroglobulin is a poor diagnostic biomarker of malignancy in follicular and Hurthle-cell neoplasms of the thyroid. Am J Surg 200, 41-46 (2010).

20. Latrofa, F. et al. Lymphocytic thyroiditis on histology correlates with serum thyroglobulin autoantibodies in patients with papillary thyroid carcinoma: impact on detection of serum thyroglobulin. J Clin Endocrinol Metab 97, 2380-2387 (2012).

21. McLeod, D. S. et al. Prognosis of differentiated thyroid cancer in relation to serum thyrotropin and thyroglobulin antibody status at time of diagnosis. Thyroid 24, 35-42 (2014).

22. Oltmann, S. C. et al. Markedly elevated thyroglobulin levels in the preoperative thyroidectomy patient correlates with metastatic burden. J Surg Res 187, 1-5 (2014).

23. Schlumberger, M. et al. Circulating thyroglobulin and thyroid hormones in patients with metastases of differentiated thyroid carcinoma: relationship to serum thyrotropin levels. J Clin Endocrinol Metab 51, 513-519 (1980).

24. Petric, R., Besic, H. \& Besic, N. Preoperative serum thyroglobulin concentration as a predictive factor of malignancy in small follicular and Hurthle cell neoplasms of the thyroid gland. World J Surg Oncol 12, 282 (2014).

25. Herle, A. J. \& Uller, R. P. Elevated serum thyroglobulin. A marker of metastases in differentiated thyroid carcinomas. J Clin Invest 56, 272-277 (1975).

26. Grimes, D. A. \& Schulz, K. F. Refining clinical diagnosis with likelihood ratios. Lancet 365, 1500-1505 (2005).

27. Kim, T. H. et al. Triage of patients with AUS/FLUS on thyroid cytopathology: effectiveness of the multimodal diagnostic techniques. Cancer Med 5, 769-777 (2016).

28. Nixon, I. J. et al. The impact of distant metastases at presentation on prognosis in patients with differentiated carcinoma of the thyroid gland. Thyroid 22, 884-889 (2012).

29. Kim, M. et al. Comparison of Thyroglobulin Measurements Using Three Different Immunoassay Kits: A BRAMHS Tg-Plus RIA Kit, a BRAMHS hTg Sensitive Kryptor Kit, and a Beckman Coulter ACCESS Immunoassay Kit. Endocrinol Metab (Seoul) 31, 462-468 (2016).

30. Altman, D. G. \& Bland, J. M. Diagnostic tests 2: Predictive values. BMJ 309, 102 (1994).

\section{Acknowledgements}

This work was supported by Samsung Medical Center (Grant no. CRO113031 to S.W.K.). We would like to thank statistician Insuk Sohn, PhD, of Samsung Biomedical Research Institute for his assistance with statistical analysis.

\section{Author Contributions}

All authors contributed to the design of the study and writing of the manuscript. Conceptualization, T.H.K., S.W.K. and J.H.C.; Resource, J.H.C., J.H.K. and J.S.K.; Data collection, Y.N.K., H.I.K. and S.Y.P.; Writing, H.K. All authors reviewed the manuscript.

\section{Additional Information}

Competing Interests: The authors declare that they have no competing interests.

Publisher's note: Springer Nature remains neutral with regard to jurisdictional claims in published maps and institutional affiliations. 
(i) Open Access This article is licensed under a Creative Commons Attribution 4.0 International License, which permits use, sharing, adaptation, distribution and reproduction in any medium or format, as long as you give appropriate credit to the original author(s) and the source, provide a link to the Creative Commons license, and indicate if changes were made. The images or other third party material in this article are included in the article's Creative Commons license, unless indicated otherwise in a credit line to the material. If material is not included in the article's Creative Commons license and your intended use is not permitted by statutory regulation or exceeds the permitted use, you will need to obtain permission directly from the copyright holder. To view a copy of this license, visit http://creativecommons.org/licenses/by/4.0/.

(C) The Author(s) 2017 